\title{
Attention Improves Population-Level Frequency Tuning in Human Auditory Cortex
}

\author{
Hidehiko Okamoto, ${ }^{1 \star}$ Henning Stracke, ${ }^{1 \star}$ Carsten H. Wolters, ${ }^{1}$ Frank Schmael, ${ }^{2}$ and Christo Pantev ${ }^{1}$ \\ ${ }^{1}$ Institute for Biomagnetism and Biosignalanalysis, and ${ }^{2}$ Department of Otorhinolaryngology, Head and Neck Surgery, University Hospital, University of \\ Muenster, 48149 Muenster, Germany
}

\begin{abstract}
Attention improves auditory performance in noisy environments by either enhancing the processing of task-relevant stimuli ("gain"), suppressing task-irrelevant information ("sharpening"), or both. In the present study, we investigated the effect of focused auditory attention on the population-level frequency tuning in human auditory cortex by means of magnetoencephalography. Using complex stimuli consisting of a test tone superimposed on different band-eliminated noises during active listening or distracted listening conditions, we observed that focused auditory attention caused not only gain, but also sharpening of frequency tuning in human auditory cortex as reflected by the $\mathrm{N} 1 \mathrm{~m}$ auditory evoked response. This combination of gain and sharpening in the auditory cortex may contribute to better auditory performance during focused auditory attention.
\end{abstract}

Key words: attention; gain; sharpening; lateral inhibition; human auditory cortex; magnetoencephalography; MEG

\section{Introduction}

The ability to encode specific sounds in noisy environments is important in daily life. In most day-to-day situations, we are exposed to many different types of sound signals simultaneously. Nevertheless, we can easily perceive selected sound signals simply by paying attention to them. The processing might be tuned by both enhancement of neural responses corresponding to taskrelevant stimuli (gain) and suppression of task-irrelevant neural activities (sharpening). Despite extensive research, the tuning effects of attention in the human auditory cortex remain to be exactly determined (Alain and Arnott, 2000).

A functional magnetic resonance imaging (fMRI) study (Murray and Wojciulik, 2004) of the visual system showed that attention not only increased the activation (gain), but also enhanced the selectivity of the neural population representing an attended object. In a manner comparable with the visual system, auditory attention might cause an overall increase in auditory neural activity (gain) and also increase the resolution of the tonotopic map, contributing to finer neural population-level coding for attended sound signals (sharpening), as illustrated in Figure $1 \mathrm{~A}$. The gain functions of attention in the human auditory system have also been investigated using fMRI and other neuroimaging techniques (Grady et al., 1997; Benedict et al., 1998; Murray and Wojciulik, 2004). Previous electroencephalography (EEG) (Hill-

\footnotetext{
Received Feb. 15, 2007; revised Aug. 3, 2007; accepted Aug. 6, 2007.

This work was supported by the Deutsche Forschungsgemeinschaft Pa 392/10-2. We thank Andreas Wollbrink for technical assistance and Claude Alain, Markus Junghoefer, and Patrick Bermudez for reading this manuscript and for helpful comments.

*H.O. and H.S. contributed equally to this work.

Correspondence should be addressed to Dr. Christo Pantev, Institute for Biomagnetism and Biosignalanalysis, Westfalian Wilhelms-University Muenster, Malmedyweg 15, 48149 Muenster, Germany. E-mail: pantev@uni-muenster.de.

D0I:10.1523/JNEUROSCI.2963-07.2007

Copyright $\odot 2007$ Society for Neuroscience $\quad$ 0270-6474/07/2710383-08\$15.00/0
}

yard et al., 1973; Picton and Hillyard, 1974) and magnetoencephalography (MEG) (Woldorff et al., 1993) studies also observed that focused auditory attention increased the N1 auditory response, a component thought to originate in lateral aspects of Heschl's gyrus and the posterior temporal plane (Pantev et al., 1995; Eggermont and Ponton, 2002). A sharpening effect of attention, however, has not yet been shown in this area.

The effect of the efferent (top-down) neural system on frequency tuning has been studied at single-neuron level. Polley et al. (2006) investigated whether bottom-up sensory inputs or topdown task-dependent processes controlled cortical reorganization in adult rats. Between two groups of rats, the authors used identical auditory stimuli, but different attention tasks. The results showed that only top-down signals played an important role in the reorganization of primary and secondary auditory cortex and, thus, attention focused on frequency cues might also intensify efferent neural inputs and expand the representation of the target frequency range within the cortical tonotopic map.

Based on the aforementioned results, the major goal of the present study was to investigate gain as well as sharpening effects of focused auditory attention on the population-level frequency tuning in human auditory cortex by means of MEG. We posit that attention might strengthen not only excitatory neural connections, but also inhibitory networks (see Fig. $1 A$ ), which would contribute to finer frequency tuning and better auditory performance.

\section{Materials and Methods}

Subjects

Participants were 13 healthy subjects between 22 and 28 years of age (seven females; mean, 24.2 years) with no history of psychological or neurological disorders. All subjects were right handed (assessed with the Edinburgh Handedness Inventory) and their hearing thresholds were within norms for the frequency range of $250-8000 \mathrm{~Hz}$, as tested by means of clinical pure tone audiometry. Participants gave written in- 
formed consent for participation in the study in accordance with procedures approved by the Ethics Commission of the Medical Faculty, University of Muenster.

\section{MEG measurement}

Experimental design and stimuli. To evaluate gain and sharpening effects of attention, we presented a test stimulus (TS) either independently or simultaneously with four different band-eliminated noises (BENs) queued in a random sequence. Neuronal activities evoked by BEN and TS can be divided into three categories: activity evoked exclusively by BEN, activity evoked exclusively by TS, or activity evoked by both BEN and TS (Fig. $1 B$, light gray, dark gray, black areas, respectively). Activities of auditory neurons, which can be activated by both BEN and TS, decrease with BEN becoming wider and/or with frequency tuning becoming sharper. Thus, the diminution of overlapping areas (Fig. $1 B$, black areas) and the enlargement of areas activated solely by TS (Fig. 1B, dark gray areas) illustrate improved population-level frequency tuning. In this study, neurons in areas of overlap (Fig. $1 B$, black areas) could be activated by both BEN and TS, but in fact they were already activated by BEN when TS was presented (Fig. 2A). Thus, the N1m response measured in this study reflects the activity of the neural group activated solely by TS onset (Fig. $1 B$, dark gray areas). If the effect of attention were gain only, the ratios of TS-related neural activities evoked in the case of each BEN condition (Fig. $1 B$, dark gray areas) compared with no BEN (Fig. $1 B$, dark gray and black areas) would not differ between active versus distracted listening conditions. In contrast, if attention sharpened the population-level frequency tuning, the ratio would become larger in the active compared with the distracted listening condition. Thus, the combination BEN plus TS allows us to measure population-level frequency tuning of the alert human auditory cortex by means of MEG.

The TS was a $40 \mathrm{~Hz} 100 \%$ amplitudemodulated tone ( $12.5 \mathrm{~ms}$ rise and fall time) with a carrier frequency of $1000 \mathrm{~Hz}$ and a duration of $0.7 \mathrm{~s}$. The sound onset asynchrony between two subsequent TS was $3.0 \mathrm{~s}$. In $10 \%$ of the trials, the TS deviated in structure from the standard TS. In these "deviant" trials, the TS contained a silent period of $50 \mathrm{~ms}$ duration ("temporal gap," $12.5 \mathrm{~ms}$ fall and rise time) starting randomly at 50,100,150, 200, 250, $300,350,400,450,500,550$, or $600 \mathrm{~ms}$ after TS onset. The deviant stimuli served to control for subject's compliance. Deviant stimuli could not be analyzed appropriately because of low signal-to-noise ratio as a result of few trials and contamination by artifacts, and were therefore excluded from additional analysis.

BENs were prepared as follows: spectral frequency bands with widths of either $20 \mathrm{~Hz}$ (BEN20), $40 \mathrm{~Hz}$ (BEN40), $80 \mathrm{~Hz}$ (BEN80), or $160 \mathrm{~Hz}$ (BEN160) centered around the $1000 \mathrm{~Hz}$ TS carrier frequency (Fig. $2 \mathrm{~B}$ ) were eliminated from $8000 \mathrm{~Hz}$ low-pass filtered white noise (corresponding to the upper frequency limit of the sound delivery system). All BENs (duration of $3.0 \mathrm{~s}$ with $12.5 \mathrm{~ms}$ rise and fall times) were presented starting $2.0 \mathrm{~s}$ before TS onset and ending $0.3 \mathrm{~s}$ after termination of TS (Fig. 2 A). All sound stimuli were prepared as sound

$\mathrm{B} 2$
Attentional Modulation of Frequency Tuning

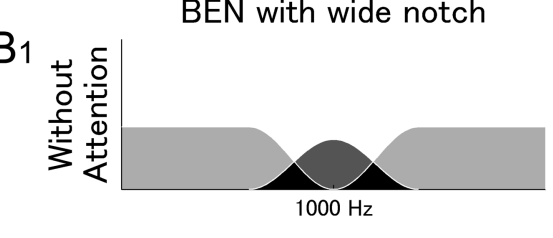

BEN with narrow notch
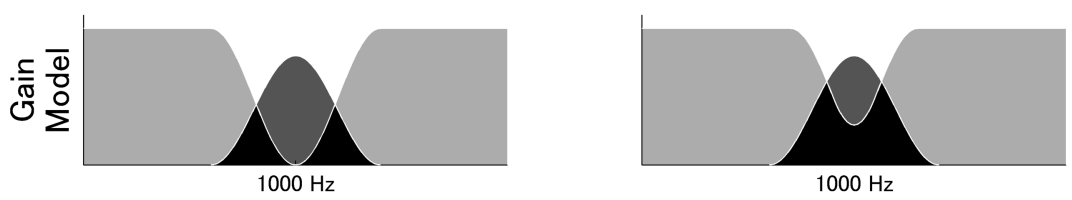

$1000 \mathrm{~Hz}$
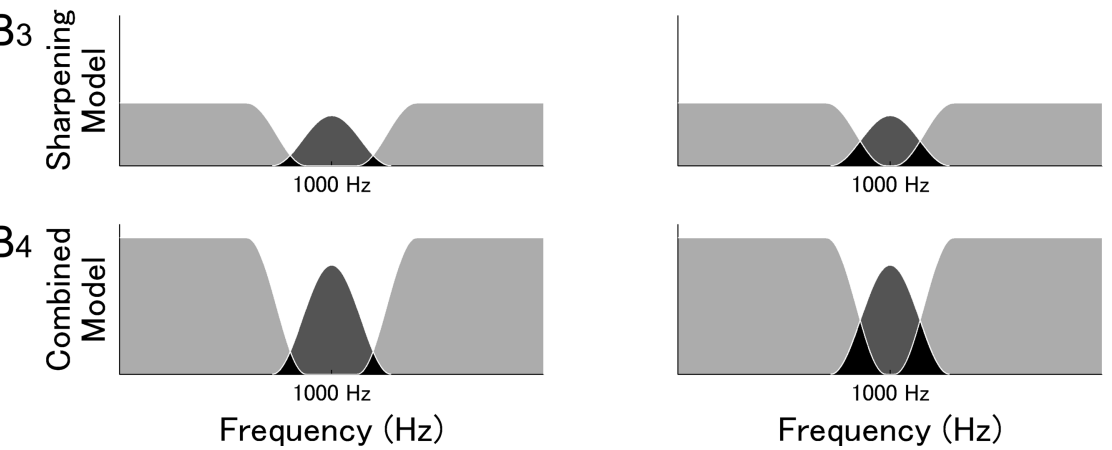

Figure 1. Attentional modulation of frequency tuning. $A$, The figure illustrates how different effects of attention [gain model vs sharpening model vs combined (gain plus sharpening) model] would modulate population-level neural activities corresponding to $00 \mathrm{~Hz}$ test stimulus. Gain is reflected by increased amplitudes, sharpening is reflected by narrow frequency distributions. . Light gray areas represent neural activities exclusively elicited by BEN, and dark gray areas represent neural activities enced by TS. Black areas indicate overlap: neurons in these areas could be activated by both BEN and TS but in fact had already been activated by BEN when TS appeared. Dark gray areas represent N1m source strength reflecting TS onset. $\boldsymbol{B}_{\boldsymbol{1}}$ displays al activities evoked without focused auditory attention (i.e., broad frequency tuning and weak neural activities as indicated by neural activities as indicated by rather wide frequency distributions and rather large amplitudes). $\boldsymbol{B}_{3}$ illustrates the sharpather small amplitudes). $\boldsymbol{B}_{4}$ displays the combined (gain plus sharpening) model (i.e., sharp frequency tuning and strong neural indicated by rather narrow frequency distributions and rather large amplitudes). Left diagrams illustrate BENs with combined models are enhanced because of the gain effect of attention. In addition, size ratios of dark gray areas between narrow BEN and wide BEN differ between models: for $\boldsymbol{B}_{\mathbf{3}}$ and $\boldsymbol{B}_{\mathbf{4}}$, ratios are much closer to 1 compared with $\boldsymbol{B}_{\mathbf{1}}$ and $\boldsymbol{B}_{\mathbf{2}}$, reflecting the sharpening effect of attention on population-level frequency tuning.

files and presented via Presentation (Neurobehavioral Systems, Albany, CA). Between BENs, there were silent intervals of $40 \mathrm{~ms}$ duration because of time delays produced by the sound presentation system. Frequency tags of $18000 \mathrm{~Hz}$ (which were not perceivable by the subject) were attached to the head of the TS to obtain precise timing of the sound stimulation. SRM-212 electrostatic earphones (Stax, Saitama, Japan) were used as transducers. Sounds were delivered through $60 \mathrm{~cm}$ silicon tubes with an inner diameter of $5 \mathrm{~mm}$ and terminating at silicon earpieces fitting to the subject's ears. The hearing threshold for the TS was determined for each individual and for each ear at the beginning of the MEG session. The TS was presented binaurally at an intensity of $35 \mathrm{~dB}$ above individual sensation level; corresponding sound pressure levels varied between 41 and $51 \mathrm{~dB}$ 

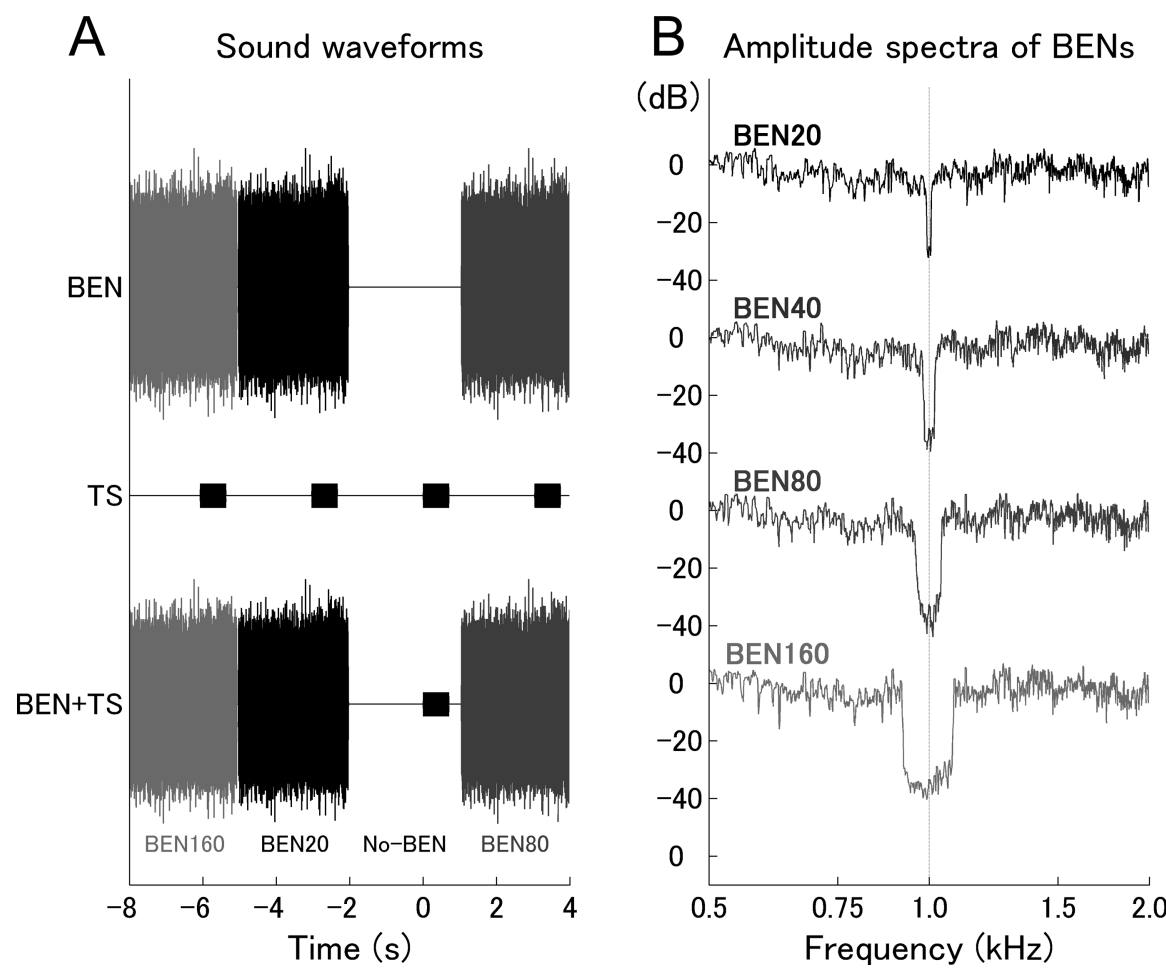

Figure 2. Experimental design. $A$, Schematic representation of the stimulation sequence. BENs of 3 s duration and the TS of 700 ms duration are displayed individually in the top and middle rows, respectively. The combined stimulation sequence (BEN + TS) is displayed in the bottom row. Note that the TS waveform is not clearly visible in the combined waveform in the bottom row because of its $15 \mathrm{~dB}$ lower amplitude compared with BEN power. $\boldsymbol{B}$, Amplitude spectra of the $3 \mathrm{~s}$ BENs measured at the earpiece. The eliminated bandwidths are $20 \mathrm{~Hz}$ (BEN20), $40 \mathrm{~Hz}$ (BEN40), $80 \mathrm{~Hz}$ (BEN80), and $160 \mathrm{~Hz}$ (BEN160). The center frequency of the eliminated region was always $1000 \mathrm{~Hz}$, which corresponded to the carrier frequency of the TS.

(mean $\pm \mathrm{SD}, 47.7 \pm 3.7$ ). The power of all BENs, which were also presented binaurally, was $15 \mathrm{~dB}$ larger than TS power. In each session, 180 trials of the standard TS for each BEN condition were presented in randomized order.

To investigate the effects of attention, we contrasted two different attentional conditions per subject: active listening and distracted listening. During active listening, subjects were instructed to focus their attention on the auditory stimuli and were required to press a response button as quickly as possible with their left or right index finger (randomized between subjects) for each detection of deviant TS. During distracted listening, no task was required; subjects watched a silent movie of their choice. The movie served to distract attention from the auditory modality. We decided not to present the silent movie during active listening because, despite clear instructions, the movie might have distracted attention from the auditory stimuli which, in turn, might have resulted in less contrast in the evoked response between the two different attentional states (Suzuki et al., 2005). The sound stimulation was identical between the two sessions, which were performed on different days. Session order was balanced across subjects.

Data acquisition and analysis. Auditory evoked fields (AEFs) were measured with a helmet-shaped 275-channel whole-head neurogradiometer (Omega; CTF Systems, Coquitlam, British Columbia, Canada) in a silent magnetically shielded room. Participants were comfortably seated upright. Head position was fixed with pads and subjects were instructed not to move. Alertness and compliance were verified via video monitoring. The measured magnetic response fields were digitally sampled at a rate of $600 \mathrm{~Hz}$. Epochs of data elicited by standard TS, including a $300 \mathrm{~ms}$ pre-TS-onset interval and a $400 \mathrm{~ms}$ post-TS-onset interval, were averaged selectively for each BEN condition after rejection of artifact epochs containing field changes larger than three picotesla. The evoked field source locations and orientations were determined in a head-based Cartesian coordinate system with the origin at the midpoint of the mediolateral axis ( $y$-axis), which joined the center points of the entrances to the ear canals (positive toward the left ear). The posterior-anterior axis ( $x$-axis) ran between nasion and origin, the inferiorsuperior axis ( $z$-axis) ran through the origin perpendicularly to the $x-y$ plane.

The N1m response is known to be generated in a relatively focused cortical area (posterior temporal plane and lateral aspects of Heschl's gyrus) (Pantev et al., 1995; Eggermont and Ponton, 2002). We estimated N1m source locations and orientations by means of two single equivalent current dipoles (one for each hemisphere) based on the no-BEN condition using a spherical head model and assuming identical locations and orientations for the BEN conditions, because a previous MEG study (Sams and Salmelin, 1994) showed that simultaneously presented BENs did not influence the calculated locations and orientations of the $\mathrm{N} 1 \mathrm{~m}$ components elicited by the test tone. For analysis of the N1m component, the averaged magnetic field signals were $30 \mathrm{~Hz}$ low-pass filtered initially followed by a baseline correction relative to the 300 ms prestimulus interval. The cortical sources were approximated individually for each subject. Initially, the time point of maximal global field power, measured as the root-mean square across all sensors around $100 \mathrm{~ms}$ after stimulus onset, was identified. Afterward, the $10 \mathrm{~ms}$ time window before the peak was used for spatiotemporal source estimation. The estimated source for each hemisphere of each subject was fixed in its location and orientation, and the source strengths were calculated for all time points and each BEN condition (BEN160, BEN80, BEN40, and BEN20). The maximal N1m source strengths were calculated in time windows between 75 and $175 \mathrm{~ms}$ (no-BEN condition), 125 and $225 \mathrm{~ms}$ (BEN 160 condition), and 150 and $250 \mathrm{~ms}$ (BEN80, BEN40, and BEN20 conditions), respectively. The estimated N1m locations with respect to each axis were evaluated by repeated-measures ANOVAs using two factors (attention: active vs distracted; hemisphere: left vs right). In this article, the $p$ values provided for repeated-measures ANOVA results are Greenhouse-Geisser corrected.

The $40 \mathrm{~Hz}$ amplitude modulated tone used as TS in this study is known to generate the so-called "auditory steady-state response" (Makela and Hari, 1987; Pantev et al., 1996; Engelien et al., 2000; Ross et al., 2000). However, it was not possible to clearly extract this response for the BEN conditions because of low signal-to-noise ratio and, thus, it was not possible to perform an auditory steady-state response analysis.

For the evaluation of the sharpening effect of attention on the population-level frequency tuning, the maximal source strength of the N1m elicited by the TS for each BEN condition in each hemisphere was normalized with respect to the maximal N1m source strength in the no-BEN condition for each subject and each hemisphere individually. Normalization was used to reduce the impact of the typically observed interindividual and intersession variability in $\mathrm{N} 1 \mathrm{~m}$ source strength. The normalization procedure was not applied to $\mathrm{N} 1 \mathrm{~m}$ latency given that the variability of latency among subjects was negligible compared with that of source strength. The normalized source strengths and latencies were then evaluated by repeatedmeasures ANOVA using three factors (BEN type: BEN160, BEN80, BEN40, and BEN20; hemisphere: left vs right; attention: active vs distracted). Post hoc comparisons were performed using BonferroniDunn's multiple-comparisons correction (significance threshold, $p<0.0083)$. In addition, non-normalized maximal N1m source strength was similarly analyzed because the hypothesized "gain" effect of attention would get lost in normalized data. 


\section{Behavioral measurement}

To evaluate the deviant detection performance of the subjects, we conducted additional behavioral measurements in a third session. These measurements took place in the MEG room and therefore stimulation devices, stimuli, and experimental parameters were identical to the "active" MEG session with the exception of likelihood of stimulus appearance: both standard and deviants appeared with a probability of 50\%. Stimulus order was pseudorandomized; each deviant stimulus was presented eight times in each BEN condition, resulting in 96 standard and 96 deviant trials for each BEN condition. Participants were instructed to press a button with their right index finger as quickly as possible when detecting a deviant stimulus. Reaction time and error rate (misses plus false alarms) were recorded. Data were analyzed with repeated-measures ANOVA (BEN type: BEN160, BEN80, BEN40, and BEN20) and post hoc comparisons were performed using Bonferroni-Dunn's multiplecomparisons correction (significance threshold, $p<0.0083$ ).

Theoretically, the possibility that participants have changed the amount of allocated attention based on BEN condition cannot completely be ruled out. In this study, subjects might have paid more attention to narrower BENs compared with wider BENs. Such strategic behavior might have been reflected by larger attentional effects for narrower compared with wider BENs. To rule out this possibility, just after the termination of the behavioral measurement, we investigated whether subjects were able to categorize the BENs with respect to task difficulty by asking the participants whether she/he had noticed that different BENs had been presented. Ten participants did not notice this at all, but three participants commented that BENs and task difficulties had differed. For these three participants, we extended the behavioral measurement to find out whether they could link BEN type to task difficulty. Participants were asked to rank BENs via button press [button 1 (easy) to button 4 (difficult)]. Each BEN was presented 15 times for $3 \mathrm{~s}$ in randomized order.

\section{Results}

\section{MEG measurement}

Clearly identifiable AEFs were obtained from all subjects in all conditions. After artifact rejection, a number of 156-180 (mean, 170) trials remained in each condition to be used for AEF averaging. Waveforms, contour maps, and estimated source localizations of the N1m elicited by no-BEN during active listening overlaid on the MRI of one representative subject are displayed in Figure 3 (supplemental Fig. 1, movies 1, 2, available at www. jneurosci.org as supplemental material). Clear dipolar patterns over the right and left hemispheres were observed. The goodnessof-fit of the underlying dipolar model for dipole estimation was in a range of $91.8-98.2 \%$ (mean $\pm \mathrm{SD}, 95.8 \pm 1.78 \%$ ), confirming the adequacy of the chosen equivalent current dipole approach. Figure 4 displays the group-averaged dipole locations of the N1m for the active and distracted listening conditions with the $95 \%$ confidence interval limits of the relative differences around the distracted listening condition.

The repeated-measures ANOVA applied to the dipole source locations of the $\mathrm{N} 1 \mathrm{~m}$ response resulted in significant main effects for hemisphere in the posterior-anterior dimension ( $x$-axis; $\left.F_{(1,12)}=5.8 ; p<0.05\right)$, the mediolateral dimension $(y$-axis; $\left.F_{(1,12)}=6.3 ; p<0.05\right)$, and the inferior-superior dimension $\left(z\right.$-axis; $\left.F_{(1,12)}=20.5 ; p<0.001\right)$. There was no significant interaction or main effect of attention. Hence, the estimated source locations of the neural activities measured differed slightly between hemispheres regardless of whether the subjects focused their attention on the stimuli or not. This asymmetric N1m location between hemispheres most likely reflects anatomical hemispheric differences (Rademacher et al., 2001). At first glance, no significant source location difference between active and distracted conditions seems to be inconsistent with previous fMRI results revealing significantly larger cortical activations during focused auditory attention (Grady et al., 1997; Benedict et al.,
A

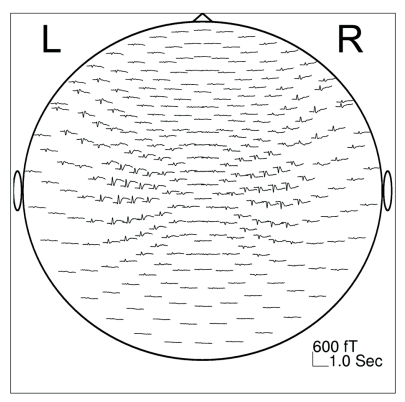

B

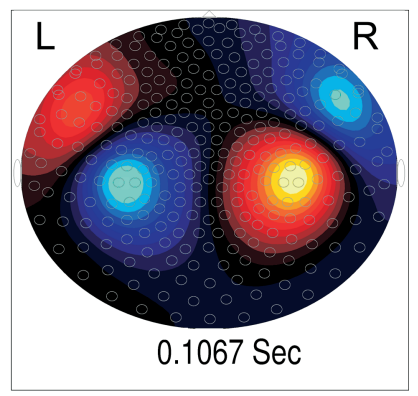

C
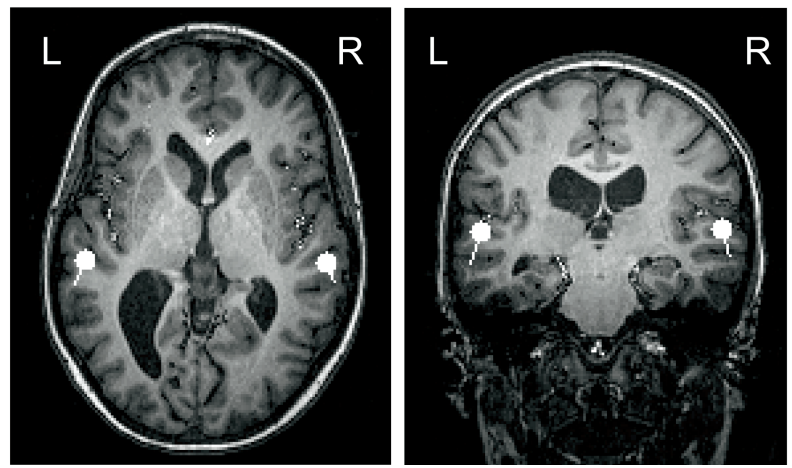

Figure 3. Representative single-subject result. $\boldsymbol{A}$, Auditory evoked magnetic fields obtained in the no-BEN condition. Thirty hertz low-pass-filtered MEG waveforms are displayed in a flattened sensor position projection. $\boldsymbol{B}$, Isocontour maps of the magnetic fields corresponding to the maximal $\mathrm{N} 1 \mathrm{~m}$ response showing dipolar patterns above both hemispheres at a latency of $0.1067 \mathrm{~s}$. Red areas represent inward flows of magnetic fields from the brain, whereas blue areas represent outward flows. C, Calculated dipole locations and orientations overlaid on an individual MRI.

1998), until it is considered that the dipole fit approach only allows estimating the center of gravity of the neural responses and not the extent of activated areas. Thus, the extent of activated neural areas may have differed between the two sessions, but the centers of gravity of the neural responses were not significantly different.

The grand-averaged N1m cortical source waveforms across all subjects (time range, -300 to $+400 \mathrm{~ms}$ ) are displayed in Figure 5. This figure demonstrates a clear N1m response peaking at $\sim 100$ $\mathrm{ms}$ after TS onset for the no-BEN condition. The N1m-responses in the BEN conditions are delayed and show smaller peaks compared with the no-BEN condition.

The averaged normalized N1m source strengths and the N1m latencies for the left and right hemispheres and each BEN condition with $95 \%$ confidence limit error bars are presented in Figure 6. The repeated-measures ANOVA applied to the normalized $\mathrm{N} 1 \mathrm{~m}$ source strengths resulted in significant main effects of BEN type $\left(F_{(3,36)}=22.8 ; p<0.0001\right)$, hemisphere $\left(F_{(1,12)}=7.4 ; p=\right.$ $0.019)$, and attention $\left(F_{(1,12)}=19.4 ; p<0.001\right)$ as well as a significant interaction between BEN type and attention $\left(F_{(3,36)}=\right.$ 5.3; $p=0.014)$. Post hoc comparisons showed significant differences between BEN160 and BEN80 ( $p<0.0003)$, BEN160 and BEN40 $(p<0.0001)$, BEN160 and BEN20 $(p<0.0001)$, and BEN80 and BEN20 $(p<0.003)$.

Moreover, because there was no significant interaction of hemisphere with any factor, we collapsed data across hemispheres and calculated planed comparisons (paired two-tailed $t$ tests; Bonferroni-Dunn-corrected significance threshold, $p<$ $0.0127)$ between active and distracted attentional states on nor- 

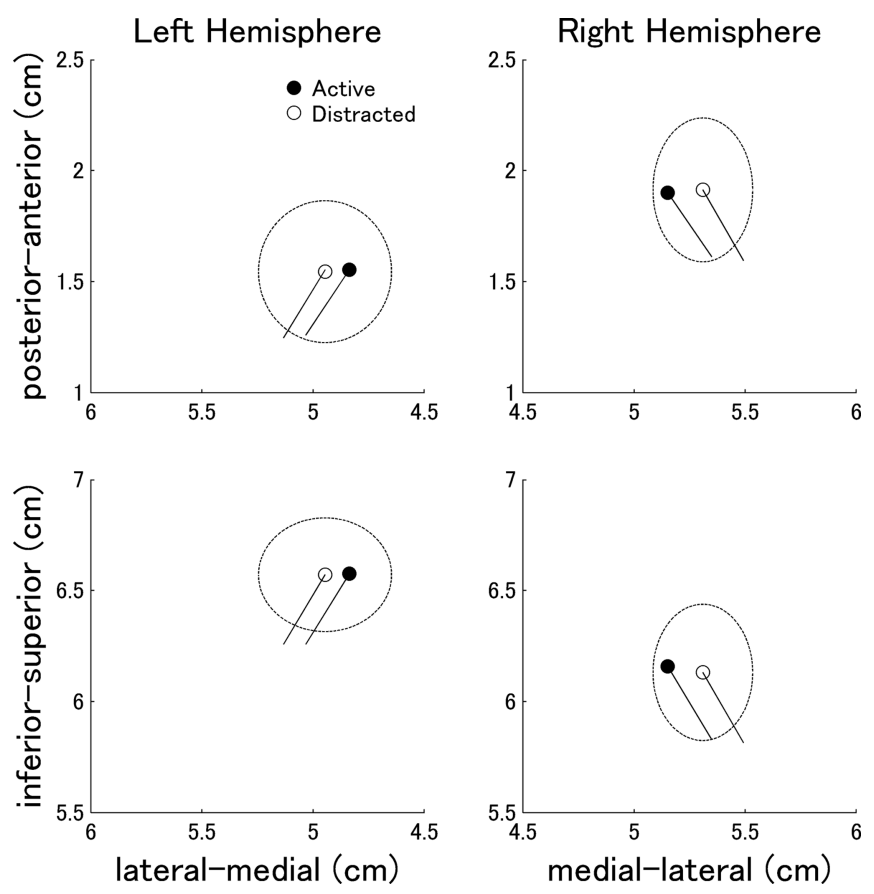

Figure 4. Estimated source locations of $\mathrm{N} 1 \mathrm{~m}$. Localization of the N1m sources in the $y-x$ plane (medial-lateral vs posterior-anterior directions) and the $y-z$ plane (medial-lateral vs inferior-superior directions). Filled symbols represent active listening; open symbols represent distracted listening. The ellipses around the open symbols denote the $95 \%$ confidence interval limits of the differences between active and distracted listening.
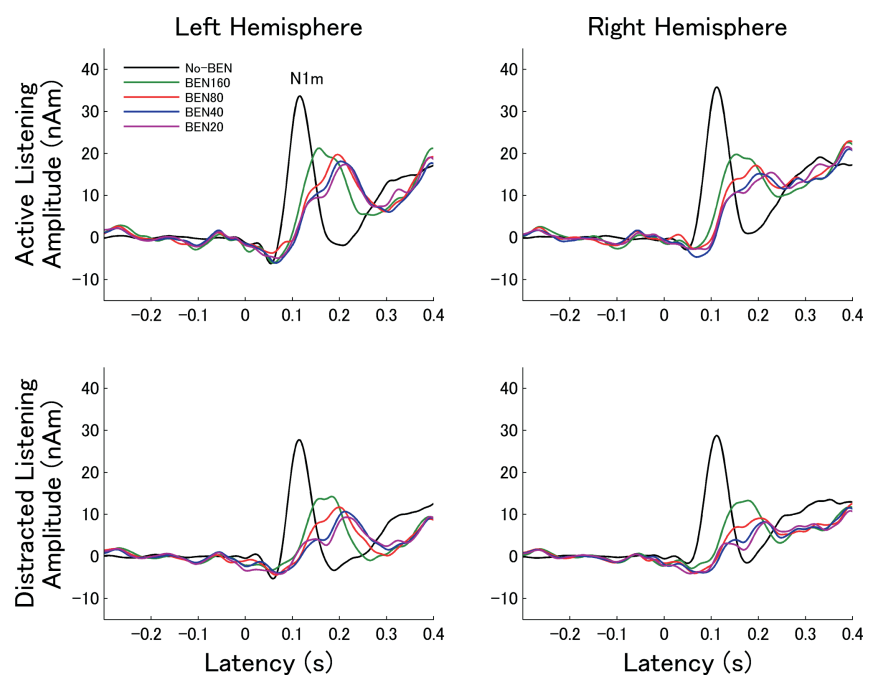

Figure 5. Grand-averaged source strength waveforms. The top graph displays grandaveraged ( $n=13$ ) source waveforms for the N1m for all BEN conditions during active listening. The bottom graph displays waveforms during distracted listening.

malized N1m source strength within each BEN condition to directly compare active and distracted conditions. The results showed significant differences between BEN160-active and BEN160-distracted $(p<0.008)$, BEN80-active and BEN80distracted $(p<0.0003)$, BEN40-active and BEN40-distracted $(p<0.0001)$ as well as between BEN20-active and BEN20distracted $(p<0.0001)$. Furthermore, we compared estimated linear slopes of change for active and distracted conditions by means of paired $t$ test. The results show that the slope for distracted was significantly steeper than the slope for active $\left(t_{(12)}=\right.$ $2.84 ; p=0.015)$.
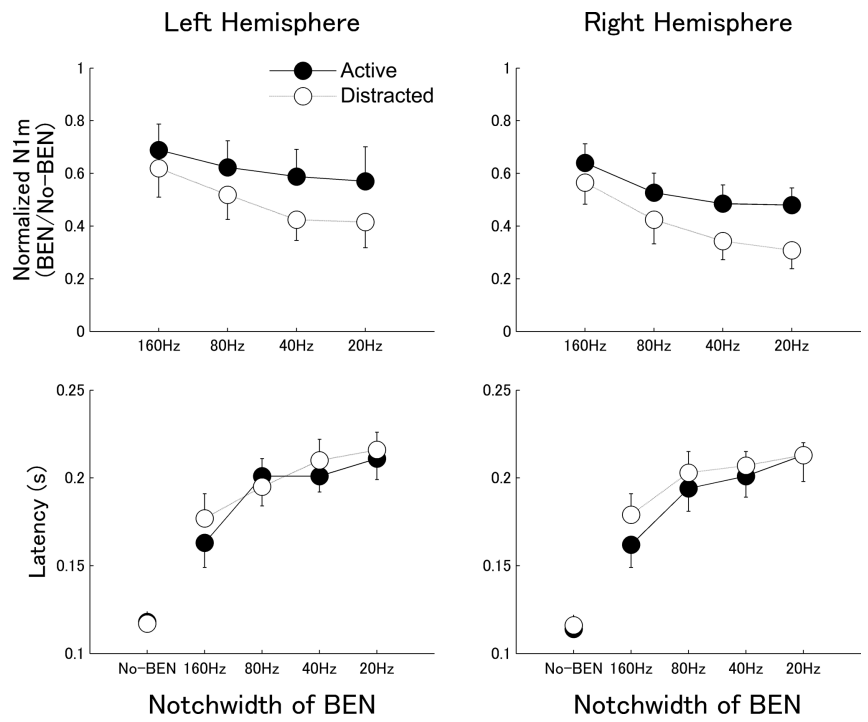

Figure 6. Normalized N1m source strengths and latencies. The graphs display the group means ( $n=13$ ) of the normalized N1m source strengths (top graphs) and latencies (bottom graphs) for each BEN condition with error bars denoting the $95 \%$ confidence intervals for the group means. Filled circles denote the responses during active listening, and open circles denote the responses during distracted listening.

Hence, statistical analyses of normalized N1m source strength indicated that attention as well as BEN type and hemisphere significantly influenced the strength of the neural activities measured. Crucially, effects of attention and BEN type were not independent from each other, but interacted: the effect of auditory focused attention increases with narrowing BEN.

The repeated-measures ANOVA applied to N1m latency revealed significant main effects of attention $\left(F_{(1,12)}=8.1 ; p=\right.$ $0.016)$ and BEN type $\left(F_{(3,36)}=63.7 ; p<0.0001\right)$, but no significant interaction between factors. Significant differences between BEN160 and all other BEN types $(p<0.0001)$ as well as between BEN80 and BEN20 ( $p<0.0001)$ were found using post hoc comparisons. Hence, both attention and BEN type influenced the timing of the neural activities measured, whereas the timing did not differ between hemispheres. The repeated-measures ANOVA applied to the non-normalized maximal N1m source strengths resulted in significant main effects for attention $\left(F_{(1,12)}=61.2\right.$; $p<0.0001)$ and BEN type $\left(F_{(3,36)}=23.0 ; p<0.0001\right)$. Thus, $\mathrm{N} 1 \mathrm{~m}$ source strength was significantly larger during active listening compared with distracted listening. Attention as well as BEN type influenced the strength of the neural activities measured. This gain effect caused by focused auditory attention did not differ between hemispheres.

\section{Behavioral measurement}

Error rates (false alarms plus misses) became larger and reaction times became longer with narrowing BENs as shown in Figure 7. The repeated-measures ANOVA applied to error rate showed a significant main effect of BEN type $\left(F_{(3,36)}=103.1 ; p<0.0001\right)$, and post hoc comparisons revealed significant differences between all BEN types $(p<0.0005)$. Also, the repeated-measures ANOVA applied to reaction time showed a significant main effect of BEN type $\left(F_{(3,36)}=46.5 ; p<0.0001\right)$, and again post hoc comparisons revealed significant differences between all BEN types $(p<0.001)$ except for BEN40 vs BEN80 $(p=0.024)$.

The results of the BEN ranking test showed that those subjects who had noticed differences between BENs were unable to rank 


\section{Behavioral Data}
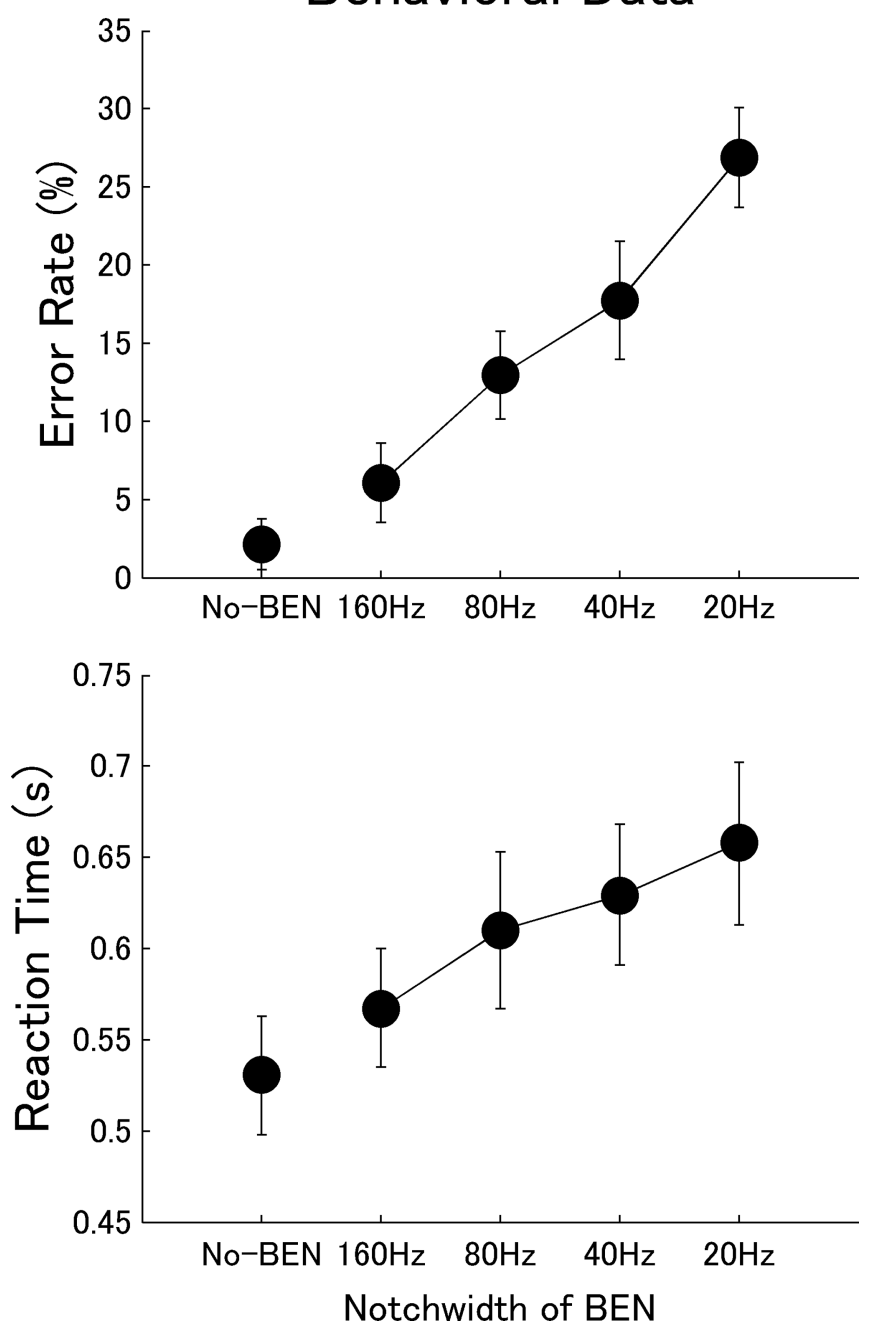

Figure 7. Behavioral measurement: error rate and reaction time. The diagrams display the error rate (top diagram) and reaction time (bottom diagram) as a function of BEN type, with error bars denoting the $95 \%$ confidence interval limits of the group $(n=13)$ means.

them properly [mean, 1 (easy) to 4 (difficult) \pm SD: BEN20, $2.62 \pm 0.41$; BEN40, $2.64 \pm 0.60$; BEN80, $2.69 \pm 0.15$; BEN160, $2.76 \pm 0.65)$. Hence, participants were not able to identify the different BENs reliably.

Moreover, to verify relationships between behavioral and electro-neurophysiological responses, we performed additional correlation analyses. For MEG variables (normalized N1m source strength during active listening and N1m latency during active listening) we obtained the means per BEN condition (BEN160, BEN80, BEN40, and BEN20) across hemispheres and subjects; for behavior variables (reaction time and error rate), we obtained the means per BEN condition across subjects. Productmoment correlations ( $\mathrm{df}=2$; critical value, 0.95 ) revealed significant relationships between normalized N1m source strength active and reaction time $(r=-0.961 ; p=0.039)$, N1m latency active and reaction time $(r=0.969 ; p=0.031)$, as well as a correlation trend between $\mathrm{N} 1 \mathrm{~m}$ latency active and error rate $(r=$ $0.913 ; p=0.087)$.

\section{Discussion}

The present study experimentally confirmed the hypothesis that AEFs elicited by TS depend on the type of simultaneously pre- sented BEN as well as the subject's attentional state. The results showed that $\mathrm{N} 1 \mathrm{~m}$ responses were significantly larger during active compared with distracted listening, especially when BENs with relatively narrow eliminated frequency bands were presented. Identical auditory stimuli (BENs and TS) were used during active and distracted listening conditions; the attentional state of the subjects differed between sessions. Therefore, afferent auditory inputs alone cannot explain the significant differences. Our results strongly suggest that focused auditory attention impacts the generators of the N1m, possibly via top-down neural connections.

In this study, we have successfully investigated neural population-level frequency tuning by means of MEG. Each BEN activated a neural population overlapping with the population corresponding to the TS. The degree of overlap differed between BENs (Fig. $1 B$, black areas); in the case of narrow BENs, fewer neurons were newly activated by the delayed TS onset (Fig. $1 B$, dark gray areas) compared with wide BENs. N1m source strength elicited by TS onset represents the number of newly activated neurons, which in turn reflects population-level frequency tuning, as has been shown by Sams and Salmelin (1994). Using a distracted listening condition, we replicated these results showing that wider BENs caused larger N1m amplitudes. Most importantly, in addition, we were able to demonstrate that the effect of BEN type significantly differed as a function of attention.

Previous EEG studies (Hillyard et al., 1973; Picton and Hillyard, 1974) showed significantly increased N1 responses during focused auditory attention. The authors suggested that focused auditory attention could modulate neural activities at an early stage of auditory analysis. In contrast, Naatanen et al. (1978) and Naatanen (1982) argued that the overlapping "processing negativity," a component of endogenous origin characterized by a source differing from N1 source (Woods and Clayworth, 1987), caused the N1 amplitude enlargement. In the present experiment, however, N1m location differences between active and distracted conditions were not observed, a result that has already been found previously (Fujiwara et al., 1998). Therefore, it is likely to assume that the N1m enlargement was caused by modulation of neural activities affecting transmission, analysis, and representation of stimulus information in the auditory pathway (Hansen, 1990). Moreover, normalized N1m source strength showed significant differences between the two attentional conditions, including a significant interaction between attention and BEN type: the normalized N1m source strength difference between active and distracted listening conditions became systematically larger with decreasing spectral notch (Fig. 6). These results imply that focused auditory attention did not only amplify neural activity, but also sharpened the frequency tuning in the auditory cortex.

A series of previous psychoacoustical studies (Schlauch and Hafter, 1991; Hafter et al., 1993; Hubner and Hafter, 1995) also supports this hypothesis. It could be demonstrated that reducing frequency uncertainty by presenting frequency cues leads to sharpened frequency tuning and improved tone-detection performance. In the present study, we used one single TS fixed in frequency for all conditions. Hence, frequency uncertainty was minimal, whereas frequency selectivity around TS frequency was maximal. Thereby, the present results may reflect the modulation of neurophysiological filtering by focused auditory attention around the test stimulus frequency.

Inhibitory neural interactions might play an essential role for this sharpening effect. Previous studies have shown that the classical lateral inhibition concept (von Békésy, 1967; Suga, 1995; 
Pantev et al., 2004; Okamoto et al., 2005, 2007) can account for sharpening of frequency tuning in the central auditory system. Afferent neural inputs consist not only of excitatory, but also of broadly tuned inhibitory inputs, which suppress surrounding neural activities resulting in improved spectral contrast. However, a cotuned excitatory and inhibitory neural model based on previous single-neuron studies (Wehr and Zador, 2003; Oswald et al., 2006) can also explain the sharpening effect. Those studies demonstrated that frequency tuning curves of excitatory and inhibitory inputs are similar. However, inhibitory inputs follow excitatory inputs with a few milliseconds delay. Cotuned neural activities can improve temporal coding by shortening the duration of excitatory neural activities and also induce finer frequency tuning by suppressing excitatory activity (Tan et al., 2004). Thus, attentional enhancement of cotuned neural activities could also improve frequency tuning.

In the present study, the inhibitory system, intensified by focused auditory attention, might have sharpened the populationlevel frequency tuning via the top-down auditory pathway. As a consequence, neurons corresponding to edge frequencies of the BENs might have been less activated by the initial part of the BENs during active listening because of steeper frequency tuning characteristics (Fig. $1 B$ ). Hence, relatively more neurons could be activated by the delayed TS onset because of relatively small overlap with neurons already activated by the initial part of the BENs, resulting in increased $\mathrm{N} 1 \mathrm{~m}$ source strength during active compared with distracted listening, especially in case of narrow BENs. To summarize, the intensified inhibitory system in the auditory cortex may explain the sharpening effect observed in response to attended stimuli.

Single-cell recording studies revealed that frequency tuning can be modulated by learning-induced plasticity in the inferior colliculus (Gao and Suga, 1998), medial geniculate body (Edeline and Weinberger, 1992; Lennartz and Weinberger, 1992), primary auditory cortex (Weinberger et al., 1984; Ohl and Scheich, 1996; Fritz et al., 2005), and secondary auditory cortical fields (Diamond and Weinberger, 1984). Suga et al. (2002) showed that electrical stimulations in auditory cortex could cause expanded or compressed reorganization in this area and also subcortical auditory nuclei via efferent inputs. The tuning curves of neurons either shifted toward the parameter values of behaviorally important sounds (expanded reorganization, gain) or away from those values (compressed reorganization, sharpening). Thus, attention might also modulate receptive fields of the cortex and subcortical auditory nuclei via the efferent auditory pathway.

However, the comparison between plasticity of frequency tuning observed in single-cell responses and attentional modulation of population-level frequency tuning of human auditory cortical responses might be inappropriate (Ohl and Scheich, 2005). Woldorff et al. (1993) observed significant attentional gain effects on both P1m and N1m, but the effect was larger on N1m. An fMRI-study also showed that the mesial part of the human auditory cortex is a stimulus-driven area that was always activated by a sound stimulus regardless of the subject's state of attention, whereas the activation of the lateral auditory cortex depended on the state of attention regardless of sound properties (Petkov et al., 2004). Although the lowest stage on which attentional sharpening occurs remains to be determined, these results indicated that attentional modulation of population-level neural activities might take place mainly on the cortical level. Thus, it is reasonable to assume that in the present study attention mainly modulated the inhibitory neural conductance of the lateral auditory cortex.
Furthermore, we observed significant hemispheric differences in normalized N1m source strength, which was larger for the left hemisphere during both active and distracted listening. These laterality effects cannot be explained by the mere presence of the task during active listening. Previous studies have shown that the left hemisphere may be dominant for processing requiring fine temporal resolution (Zatorre and Belin, 2001), and temporal coherence seems to be crucial for the segregation of target sounds from nontarget sounds (Barbour and Wang, 2002). Other authors (Poeppel, 2003; Boemio et al., 2005) have suggested the "asymmetric sampling in time" hypothesis. This model suggests that the left auditory cortex dominantly exploits short temporal integration windows $(20-40 \mathrm{~ms})$, whereas the right auditory cortex exploits longer ones (150-250 ms). In the present study, information from short temporal integration windows would be important for detection of the TS onset inside the BENs. Thus, the left hemispheric dominance observed here may imply that the left hemisphere plays a more important role for monitoring and analyzing auditory signals in noisy environments.

One can hypothesize that subjects may allocate more or less attention to solving a task depending on its difficulty. In this study, subjects were unable to strategically adjust the degree of attention allocated before TS onset based on BEN type. Moreover, transient and sustained AEFs elicited by the initial part of the different BENs did not differ systematically, but were generally larger in case of focused auditory attention. Sustained AEFs evoked by BENs overlapped with neural activities elicited by TS. However, by applying baseline correction, we eliminated the sustained neural activities elicited by BENs (Fig. $1 \mathrm{~B}$, black and light gray areas) and were able to successfully extract neural responses elicited by the test stimulus only (dark gray areas).

In conclusion, this study has shown for the first time that focused auditory attention cannot only amplify neural activities in general (gain effect), but also can sharpen population-level frequency tuning in the human auditory cortex, possibly via the inhibitory system. Auditory cortical neurons seem to be influenced by both: bottom-up physical sound features and top-down attentional processes. Effects of bottom-up and top-down neural inputs on excitatory and inhibitory neural networks within the human auditory cortex result in enhanced and sharpened population-level neural responses, which are reflected by the N1m response during focused auditory attention.

\section{References}

Alain C, Arnott SR (2000) Selectively attending to auditory objects. Front Biosci 5:D202-D212.

Barbour DL, Wang X (2002) Temporal coherence sensitivity in auditory cortex. J Neurophysiol 88:2684-2699.

Benedict RH, Lockwood AH, Shucard JL, Shucard DW, Wack D, Murphy BW (1998) Functional neuroimaging of attention in the auditory modality. NeuroReport 9:121-126.

Boemio A, Fromm S, Braun A, Poeppel D (2005) Hierarchical and asymmetric temporal sensitivity in human auditory cortices. Nat Neurosci 8:389-395.

Diamond DM, Weinberger NM (1984) Physiological plasticity of single neurons in auditory cortex of the cat during acquisition of the pupillary conditioned response: II. Secondary field (AII). Behav Neurosci 98:189-210.

Edeline JM, Weinberger NM (1992) Associative retuning in the thalamic source of input to the amygdala and auditory cortex: receptive field plasticity in the medial division of the medial geniculate body. Behav Neurosci 106:81-105.

Eggermont JJ, Ponton CW (2002) The neurophysiology of auditory perception: from single units to evoked potentials. Audiol Neurootol 7:71-99.

Engelien A, Schulz M, Ross B, Arolt V, Pantev C (2000) A combined func- 
tional in vivo measure for primary and secondary auditory cortices. Hear Res 148:153-160.

Fritz J, Elhilali M, Shamma S (2005) Active listening: task-dependent plasticity of spectrotemporal receptive fields in primary auditory cortex. Hear Res 206:159-176.

Fujiwara N, Nagamine T, Imai M, Tanaka T, Shibasaki H (1998) Role of the primary auditory cortex in auditory selective attention studied by wholehead neuromagnetometer. Brain Res 7:99-109.

Gao E, Suga N (1998) Experience-dependent corticofugal adjustment of midbrain frequency map in bat auditory system. Proc Natl Acad Sci USA 95:12663-12670.

Grady CL, Van Meter JW, Maisog JM, Pietrini P, Krasuski J, Rauschecker JP (1997) Attention-related modulation of activity in primary and secondary auditory cortex. NeuroReport 8:2511-2516.

Hafter ER, Schlauch RS, Tang J (1993) Attending to auditory filters that were not stimulated directly. J Acoust Soc Am 94:743-747.

Hansen JC (1990) Processing negativity-comparison process or selective processing. Behav Brain Sci 13:242.

Hillyard SA, Hink RF, Schwent VL, Picton TW (1973) Electrical signs of selective attention in the human brain. Science 182:177-180.

Hubner R, Hafter ER (1995) Cuing mechanisms in auditory signal detection. Percept Psychophys 57:197-202.

Lennartz RC, Weinberger NM (1992) Frequency-specific receptive-field plasticity in the medial geniculate-body induced by pavlovian fear conditioning is expressed in the anesthetized brain. Behav Neurosci 106:484-497.

Makela JP, Hari R (1987) Evidence for cortical origin of the $40 \mathrm{~Hz}$ auditory evoked response in man. Electroencephalogr Clin Neurophysiol 66:539-546.

Murray SO, Wojciulik E (2004) Attention increases neural selectivity in the human lateral occipital complex. Nat Neurosci 7:70-74.

Naatanen R (1982) Processing negativity—an evoked-potential reflection of selective attention. Psychol Bull 92:605-640.

Naatanen R, Gaillard AW, Mantysalo S (1978) Early selective-attention effect on evoked-potential reinterpreted. Acta Psychologica 42:313-329.

Ohl FW, Scheich H (1996) Differential frequency conditioning enhances spectral contrast sensitivity of units in auditory cortex (field $\mathrm{Al}$ ) of the alert Mongolian gerbil. Eur J Neurosci 8:1001-1017.

Ohl FW, Scheich H (2005) Learning-induced plasticity in animal and human auditory cortex. Curr Opin Neurobiol 15:470-477.

Okamoto H, Kakigi R, Gunji A, Kubo T, Pantev C (2005) The dependence of the auditory evoked $\mathrm{N} 1 \mathrm{~m}$ decrement on the bandwidth of preceding notch-filtered noise. Eur J Neurosci 21:1957-1961.

Okamoto H, Kakigi R, Gunji A, Pantev C (2007) Asymmetric lateral inhibitory neural activity in the auditory system: a magnetoencephalographic study. BMC Neurosci 8:33.

Oswald AMM, Schiff ML, Reyes AD (2006) Synaptic mechanisms underlying auditory processing. Curr Opin Neurobiol 16:371-376.

Pantev C, Bertrand O, Eulitz C, Verkindt C, Hampson S, Schuierer G, Elbert $\mathrm{T}$ (1995) Specific tonotopic organizations of different areas of the human auditory cortex revealed by simultaneous magnetic and electric recordings. Electroencephalogr Clin Neurophysiol 94:26-40.

Pantev C, Roberts LE, Elbert T, Ross B, Wienbruch C (1996) Tonotopic organization of the sources of human auditory steady-state responses. Hear Res 101:62-74.

Pantev C, Okamoto H, Ross B, Stoll W, Ciurlia-Guy E, Kakigi R, Kubo T (2004) Lateral inhibition and habituation of the human auditory cortex. Eur J Neurosci 19:2337-2344.

Petkov CI, Kang X, Alho K, Bertrand O, Yund EW, Woods DL (2004) Attentional modulation of human auditory cortex. Nat Neurosci 7:658-663.

Picton TW, Hillyard SA (1974) Human auditory evoked potentials. II. Effects of attention. Electroencephalogr Clin Neurophysiol 36:191-199.

Poeppel D (2003) The analysis of speech in different temporal integration windows: cerebral lateralization as "asymmetric sampling in time." Speech Commun 41:245-255.

Polley DB, Steinberg EE, Merzenich MM (2006) Perceptual learning directs auditory cortical map reorganization through top-down influences. J Neurosci 26:4970-4982.

Rademacher J, Morosan P, Schormann T, Schleicher A, Werner C, Freund HJ, Zilles K (2001) Probabilistic mapping and volume measurement of human primary auditory cortex. NeuroImage 13:669-683.

Ross B, Borgmann C, Draganova R, Roberts LE, Pantev C (2000) A highprecision magnetoencephalographic study of human auditory steadystate responses to amplitude-modulated tones. J Acoust Soc Am 108:679-691.

Sams M, Salmelin R (1994) Evidence of sharp frequency tuning in the human auditory cortex. Hear Res 75:67-74.

Schlauch RS, Hafter ER (1991) Listening bandwidths and frequency uncertainty in pure-tone signal detection. J Acoust Soc Am 90:1332-1339.

Suga N (1995) Sharpening of frequency tuning by inhibition in the central auditory system: tribute to Yasuji Katsuki. Neurosci Res 21:287-299.

Suga N, Xiao Z, Ma X, Ji W (2002) Plasticity and corticofugal modulation for hearing in adult animals. Neuron 36:9-18.

Suzuki J, Nittono H, Hori T (2005) Level of interest in video clips modulates event-related potentials to auditory probes. Int J Psychophysiol 55:35-43.

Tan AY, Zhang LI, Merzenich MM, Schreiner CE (2004) Tone-evoked excitatory and inhibitory synaptic conductances of primary auditory cortex neurons. J Neurophysiol 92:630-643.

von Békésy G (1967) Sensory inhibition. Princeton, NJ: Princeton UP.

Wehr M, Zador AM (2003) Balanced inhibition underlies tuning and sharpens spike timing in auditory cortex. Nature 426:442-446.

Weinberger NM, Hopkins W, Diamond DM (1984) Physiological plasticity of single neurons in auditory cortex of the cat during acquisition of the pupillary conditioned response. I. Primary field (AI). Behav Neurosci 98:171-188.

Woldorff MG, Gallen C, Hampson SA, Hillyard SA, Pantev C, Sobel D, Bloom FE (1993) Modulation of early sensory processing in human auditory cortex during auditory selective attention. Proc Natl Acad Sci USA 18:8722-8726.

Woods DL, Clayworth CC (1987) Scalp topographies dissociate N1 and Nd components during auditory selective attention. Electroencephalogr Clin Neurophysiol 40:155-160.

Zatorre RJ, Belin P (2001) Spectral and temporal processing in human auditory cortex. Cereb Cortex 11:946-953. 